\title{
Spectroscopic Investigation of Increased Fluorescent Intensity of Fluorescent Dyes When Adsorbed onto Polystyrene Microparticles
}

\author{
Hiroshi AOKI, ${ }^{\dagger}$ Masaki Torimura, and Hiroshi HABE \\ National Institute of Advanced Industrial Science and Technology (AIST), 16-1 Onogawa, Tsukuba, \\ Ibaraki 305-8569, Japan
}

\begin{abstract}
Microplastics as environmental pollutants are increasingly a source of alarm. The characterization of microplastics will be necessary to discriminate microplastics from other types of particles. To discriminate specific microplastics, plasticadsorbable fluorescent dyes are used, the stained microplastics are separated from the dye-microplastic mixture by filtration, and the type of fluorescent staining of the microplastics is analyzed by fluorescent microscopy. In this study, to realize the in situ analysis of fluorescent staining, i.e., to discriminate microplastics without any separation or filtration processes, we studied the change in the fluorescent properties after adsorption of the fluorescent dyes to the microplastic particle surfaces using a 3D excitation emission matrix fluorescence spectroscopy (the excitation wavelength-dependent emission spectrum). We used three fluorescent dyes: Fluorescein, Rhodamine 6G, and Methylene Blue, and polystyrene microparticles as our model microplastic. Fluorescein and Methylene Blue showed increases in the fluorescent intensity, while Rhodamine $6 \mathrm{G}$ showed negligible intensity changes. This is likely due to the degree of affinity of the dyes to the polystyrene particle surface, the structural stability of the dyes on the surface, and the changes in the environment around the dyes after the adsorption of each dye to the surface. We conclude that we have demonstrated the potential to look for appropriate fluorescent dyes using the method studied here to identify and estimate individual plastic materials.
\end{abstract}

Keywords Microplastics, nanoplastics, fluorescence, surface, binding, spectra, detection, analysis

(Received November 30, 2020; Accepted February 11, 2021; Advance Publication Released Online by J-STAGE February 19, 2021)

\section{Introduction}

Human beings have produced vast quantities of chemical substances as part of their continuing economic progress and improvement of quality of life. ${ }^{1,2}$ These chemical substances have proved to be mostly beneficial and convenient; however, some of them, when released into the environment, risk becoming chemical hazards that threaten our lives. ${ }^{2}$ Chemical substances intrinsically carry the risk of acting as sources of pollutants. It is necessary to study the physical, chemical, and biological characteristics of such chemical substances, including their toxicity and physiological and ecological effects, to be able to make the best use of them..$^{3,4}$

Over the last decade, microplastics have been increasingly understood to be potential environmental pollutants. ${ }^{5}$ It is suspected that microplastics act as vectors for various toxic chemicals, and that they may therefore cause imbalances in ecosystems and affect food chains. ${ }^{67}$ Microplastics are very common. Medicines and cosmetics contain microplastics to enhance and improve their functions. ${ }^{89}$ Synthetic fabrics produce microplastics whenever they are washed. ${ }^{10}$ Wasted plastics also shed microplastics. ${ }^{11}$ Although microplastics need to be observed microscopically due to their diameters being from a few $\mu \mathrm{m}$ to $5 \mathrm{~mm},{ }^{12}$ it is difficult to understand their nature because they are made of various polymer materials or

† To whom correspondence should be addressed.

E-mail: aoki-h@aist.go.jp mixtures of them, and are damaged when subjected to UV light, heat, high humidity, and pressure. ${ }^{13}$ Further progress is therefore needed in the development of techniques to characterize them.

When identifying microplastics, it is important to discriminate them from other particles, such as mineral-originated particles, like silica and mica, biological-originated particles including tissue and bone fragments, and between microplastics made of other polymer materials. Researchers currently use plasticadsorbable fluorescent dyes to identify particular microplastics. The microplastics are mixed with the dyes, the microplastics are rinsed with solvents, and the stained microplastics are then identified by analyzing their fluorescence by means of fluorescence spectroscopy. ${ }^{13-19}$ However, the washing process used to separate the stained microplastics from unbound dyes is laborious and shows the throughput of microplastic analyses. This prompted us to attempt to improve the throughput by enabling analysis of the fluorescent staining of the microplastics in the mixture with the dyes in situ, without the need for a washing process.

Some fluorescent dyes change their fluorescence characteristics under particular conditions. This is well known as the working principle of molecular beacons, in which a reporter fluorescent dye, e.g. , Fluorescein, is quenched by a proximal quencher, e.g., Dabcyl (4-((4-(dimethylamino)phenyl)azo)benzoic acid). ${ }^{20-22}$ This is because the energy of the fluorescent emissions of Fluorescein transfers to Dabcyl as thermal energy, resulting in the quenching of Fluorescein. Fluorescent intercalators, such as SYBR $^{\circledR}$ Green (Thermo Fisher Scientific; Tokyo, Japan), enhance and shift the fluorescence to intercalate minor grooves 
in double-stranded DNA molecules. ${ }^{23}$ This is because the restriction of the structural mobility of the dye causes this energy to be released as fluorescence.

If these changes in the fluorescent properties of the dyes upon binding to the plastic surfaces vary according to the plastic material used, we can expect to realize an in situ analysis of fluorescent staining to discriminate microplastics without any separation and filtration processes. If the changes in the fluorescent property of the dyes depends on the materials comprising the microplastics, it should be possible to identify the material that they are made of.

In this study, to establish a strategy to search for fluorescent dyes whose fluorescent properties change upon adsorption to a material's surface, we used commercially available fluorescent dyes and microplastic particles, and investigated any changes in the fluorescent properties of the dyes using a 3D excitation emission matrix (EEM) fluorescence spectroscopy (investigation of the excitation wavelength-dependent emission spectrum). We used three fluorescent dyes (Fluorescein, Rhodamine 6G, and Methylene Blue) and polystyrene microparticles as our model microplastic. Fluorescein and Methylene Blue showed a large change in the fluorescent intensity both before and after adding the particles. We concluded this to be due to the interaction between the fluorescent dyes and the polystyrene particle surface, and also due to the environment around the dyes changing from a hydrophilic environment of the bulk of aqueous solution to a hydrophobic environment of the polystyrene surface.

\section{Experimental}

\section{Reagents}

The microplastics used in this study were SPHERO Ultra Rainbow Fluorescent Particles URFP-100 (diameter, 8.0 $12.9 \mu \mathrm{m}$; concentration, $1 \times 10^{7}$ particles $/ \mathrm{mL}$ ) and URFP-30 (diameter, 3.0 - $3.4 \mu \mathrm{m}$; concentration, $1 \times 10^{7}$ particles $/ \mathrm{mL}$ ), and Latex Microsphere Suspension 5320A (LPS32; polystyrene particle; average diameter, $3.2 \mu \mathrm{m}$; coefficient of variation, $\leq 5 \%$; concentration, $10 \%$ solids by weight $(\mathrm{w} / \mathrm{w})$; density, $1.05 \mathrm{~g} / \mathrm{mL}$ ) from Thermo Fisher Scientific. Phosphate-buffered saline (PBS) was prepared from a 10-fold dilution of $10 \mathrm{X}$ D-PBS (100 mM phosphate, pH 7.4; Fujifilm Wako Pure Chemical, Osaka, Japan). Tween 20 (polyoxyethylene sorbitan monolaurate) was obtained from Tokyo Chemical Industry (TCI; Tokyo, Japan). The fluorescent dyes Fluorescein, Rhodamine 6G, and Methylene Blue (Fig. 1), were obtained from Fujifilm Wako. All aqueous solutions were prepared with deionized and charcoal-treated water (specified resistance $>18.2 \mathrm{M} \Omega \mathrm{cm}$ ), obtained using a Milli-Q reagentgrade water system (Merck Millipore; Bedford, MA).

\section{Preparation of samples}

The methods for preparing all of the solutions are listed in Scheme S1. Briefly, the solutions were prepared to dilute purchased particle suspensions and/or fluorescent dye stock solutions with phosphate buffered solutions. In this study, we used diluted solutions of URFP-100 and URFP-30 for basic experiments on the precipitation of particles in fluorescence spectroscopy. The working solutions of the particles were prepared by diluting the original URFP-100 and URFP-30 with $1 X$ D-PBS solutions without surfactants according to the manufacturer's instructions. The diluted solutions of LPS32 were, on the other hand, prepared by diluting the original solution of LPS32 with a 1X D-PBS solution with a $0.01 \%$ Tween 20 surfactant according to its instructions. The<smiles>O=C(O)c1ccccc1-c1c2ccc(=O)cc-2oc2cc(O)ccc12</smiles>

Fluorescein

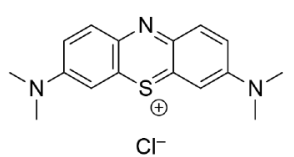

Methylene Blue<smiles>CCN=c1ccc2c(-c3ccccc3C(=O)OCC)c3cc(C)c(NCC)cc3oc-2c1</smiles>

Rhodamine 6G

Fig. 1 Molecular structures of fluorescent dyes used in this study.

concentration of Tween 20 was optimized to meet requirements for effective dispersion of particles with as little as possible use of the surfactant, and to obtain reproducible results with fluorescent spectroscopy. In experiments using fluorescent dyes, we used solutions of $50 \mathrm{nM}$ fluorescent dyes prepared from their stock solutions of 0.25 or $0.5 \mathrm{mM}$ fluorescent dye in $1 \mathrm{X}$ D-PBS solutions by diluting with a $1 \mathrm{X}$ D-PBS solution that included $0.01 \%$ Tween 20 surfactant.

\section{Particle counting}

We counted the numbers of particles in diluted solutions of LPS32 for evaluating the reproductivity of the solution preparation using a Scepter 2.0 Handheld Automated Cell Counter with a 40- $\mu \mathrm{m}$ Scepter sensor (Merck Millipore), which counts particles with diameters of 3-17 $\mu \mathrm{m}$. LPS32 has a distribution of particles with an average diameter of $3.2 \mu \mathrm{m}$ and a CV value of $5 \%$ (i.e., $3.2 \pm 0.16 \mu \mathrm{m}$ ). Although the Scepter Cell Counter cannot count particles with diameters smaller than $3 \mu \mathrm{m}$, we used them to evaluate the reproducibility of the preparation of LPS32 solutions, since the values obtained by the Scepter Cell Counter can represent total numbers of particles if the distribution of the particles is maintained during the preparation.

\section{Spectroscopic measurements}

Fluorescence spectroscopy was performed using an Aqualog fluorescence spectrometer (Horiba; Kyoto, Japan) with 3D EEM fluorescence spectroscopy software (resulting in a contour plot for excitation wavelength-dependent emission spectrum) installed in the Aqualog. The CCD gain was set at medium. The obtained data were subjected to the removal of artifacts pertaining to the inner-filter effect from the data and masking any Rayleigh scattering lines that appear in the data, according to the software instructions. The data of the contour plots for a $1 X$ D-PBS solution were subtracted from all the data of those for solutions containing fluorescent molecules and/or particles. This was followed by data analysis and discussion.

\section{Results and Discussion}

\section{Reproducible preparation of solutions}

Before starting 3D EEM fluorescence spectroscopy, the stability of microplastics in 1X D-PBS solutions was investigated using URFP-100 $(10 \mu \mathrm{m})$ and URFP-30 $(3 \mu \mathrm{m})$. According to their instructions, the concentration (numbers) of the particles in 


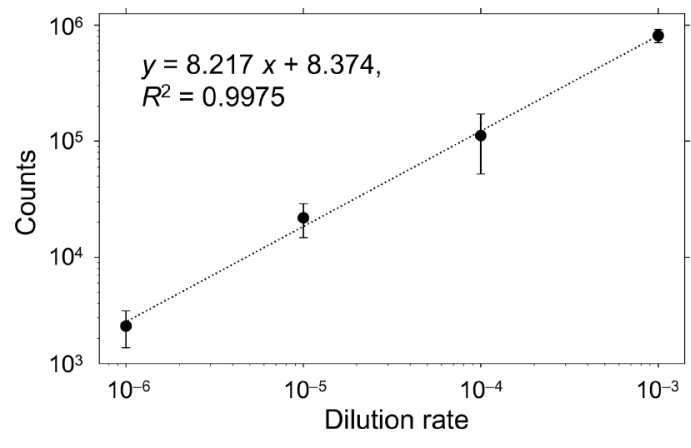

Fig. 2 Log-log plot of the relationship between the dilution rate of solutions of LPS32 particles and counts of the numbers of particles, counted using a Scepter Cell Counter with a $40-\mu \mathrm{m}$ Scepter sensor. The values of the counts here are averages for seven separate trials.

them were $1 \times 10^{7}$ particles $/ \mathrm{mL}$. The measurement of fluorescence in a $10^{3}$-times diluted solution of URFP-100 3D EEM fluorescence spectroscopy showed a decrease in the fluorescent intensity within a few tens of minutes (see Fig. S1). However, for a $10^{3}$-times diluted solution of URFP-30, the fluorescent intensity remained unchanged after $60 \mathrm{~min}$ (see Fig. S2). This is probably because larger-diameter particles (e.g., URFP-100) tend to precipitate, and the number of particles dispersed along the light becomes lower than for smaller particles (e.g., URFP-30). In following experiments, therefore, we used particles with a diameter of $3 \mu \mathrm{m}$.

The particle number of LPS32 was not instructed by the manufacture. We calculated the number as follows. According to the information provided by Thermo Fisher Scientific, LPS32 has a density of $1.05 \mathrm{~g} / \mathrm{mL}$ and a concentration of $10 \%(\mathrm{w} / \mathrm{w})$, resulting in a specific gravity of $1.5 \mathrm{~g} / \mathrm{mL}$. A sphere of LPS32 with a diameter of $3.2 \mu \mathrm{m}$ weighs $2.57 \times 10^{-11} \mathrm{~g}$. From its specific gravity of $1.5 \mathrm{~g} / \mathrm{mL}$, the weight of all the particles in $1 \mathrm{~mL}$ of LPS32 can be calculated to be $0.15 \mathrm{~g}$. We can find that the number of the particles is $5.8 \times 10^{9}$ particles $/ \mathrm{mL}$. The full details of the calculation are shown in Scheme S2.

In this study, we first evaluated the reproducibility of preparation of diluted solutions of LPS32 based on fluorescent intensity from LPS32 microparticles by calculating the deviations. We prepared 104-times diluted LPS32 solutions without using any surfactants and observed the 3D EEM fluorescence spectra of the solutions. The observed deviation (CV value) was found to be $37 \%$. When we used $0.01 \%$ Tween 20 as a surfactant, however, we observed a deviation of $4.1 \%$, a nine-fold decrease. We concluded this to be because the use of Tween 20 helps with dispersion of LPS32 in aqueous solutions. Higher concentrations (up to $1 \%$ ) of Tween 20 did not improve more the deviation. From the viewpoint that surfactants have a possibility to make the particle surface hydrophilic to hinder the adsorption of the dyes onto the surface, in the following experiments, we used $0.01 \%$ Tween 20 as a surfactant in the preparation of solutions.

To ascertain the reproducibility of the preparation of the LPS32 solutions, we counted the numbers of LPS32 particles in the solutions using a Scepter Cell Counter. As mentioned in the section of Particle counting, the Counter can count only particles with diameters of 3-17 $\mathrm{m}$. LPS32 has a distribution of particles with an average diameter of $3.2 \mu \mathrm{m}$ and a CV value of $5 \%$. When the distribution follows a normal distribution, the Counter can count $89.4 \%$ of the LPS32 particles. If the distribution of the particles is maintained while preparing the solution, the obtained particle number from the Counter is likely to be proportional to the total number of particles in the solution.

Figure 2 shows the numbers of particles counted by the Cell Counter in a log-log plot for the prepared solutions containing $10^{3}-, 10^{4}-, 10^{5}-$, and $10^{6}$-times diluted LPS32. The linear regression of the plot has a correlation coefficient, $R^{2}$, of 0.9975 , and a proportional coefficient of 8.217 , showing that the linear dilution series of the LPS32 were correctly prepared.

Spectroscopic modulation of fluorescent dyes triggered by their binding to plastic particle surfaces

Next, we investigated the changes in the fluorescent properties of the fluorescent dyes Fluorescein, Methylene Blue, and Rhodamine 6G (Fig. 1), based on the 3D EEM fluorescence spectroscopy technique when they were mixed with LPS32 particles. The concentrations of the fluorescent dyes were determined to be $50 \mathrm{nM}$. Figure $3 \mathrm{a}$ shows the results of a $3 \mathrm{D}$ EEM fluorescence spectroscopy for Fluorescein. It indicates that Fluorescein adsorbs excitation light centralizing around a wavelength of $485 \mathrm{~nm}$ and emits light centralizing around a wavelength of $513 \mathrm{~nm}$. Here, the maximum fluorescent intensity was $6479 \mathrm{AU}$. In the presence of $10^{5}$-times diluted LPS32 particles in this solution, the excitation and emission wavelengths did not change, but the intensity increased slightly (Figs. 3b and 3c). In the presence of 104-times diluted LPS32 particles in the solution, a similar pattern emerged: both of the wavelengths were almost the same as before and the intensity increased to a similar extent (Figs. $3 \mathrm{~d}$ and 3e). As a result, the fluorescent intensity for the solutions containing LPS32 particles significantly increased by up to $19.7 \%$ compared to that for solutions not containing LPS32 particles. The Welch's $t$-test also supports this significant increase in the fluorescent intensity after addition of the particles $(p=0.0077)$. The adsorption of Fluorescein to the particle surface was already thought to be saturated in the case of $10^{5}$-times diluted LPS32 particles. The excitation and emission wavelengths and the changes in the fluorescent intensities are summarized in Table 1 and Fig. 4, respectively. Here, the excitation and emission spectra might be helpful to understand the phenomena as extracts of the 3D EEM. Figure S3a shows the excitation and emission spectra at the wavelengths mentioned in Table 1, where the maximum emission and excitation signal intensities were observed, respectively.

Figure 5 shows the results of the 3D EEM spectroscopy for Methylene Blue, indicating that the excitation and emission wavelengths are 662 and $682 \mathrm{~nm}$, with a maximum fluorescent intensity of $362 \mathrm{AU}$. Here, the intensities are represented on a logarithmic scale to clearly show the intensities of Methylene Blue and LPS32 particles because of the large difference in their intensities. The addition of $10^{5}$-times diluted LPS32 particles to the solution caused an increase in the fluorescent intensity, though the excitation and emission wavelengths remained uncharged. Similarly, the addition of $10^{4}$-times diluted LPS32 particles showed the same degree of increase, indicating the adsorption of Methylene Blue to the particle surface to have been already saturated by the addition of $10^{5}$-times diluted particles. An increase in the fluorescent intensity by up to $18.2 \%$ - a significant difference-was observed as a result. The Welch's $t$-test also supports this significant increase in the fluorescent intensity after addition of the particles $(p=0.016)$. The change in the intensity after adding LPS32 particles for Methylene Blue was similar to that for Fluorescein, while the magnitude of the observed intensities for Methylene Blue was smaller than those for Fluorescein. In the same way as for Fluorescein, it is possible that the adsorption of Methylene Blue to the surface of polystyrene particle caused the observed 
(a)

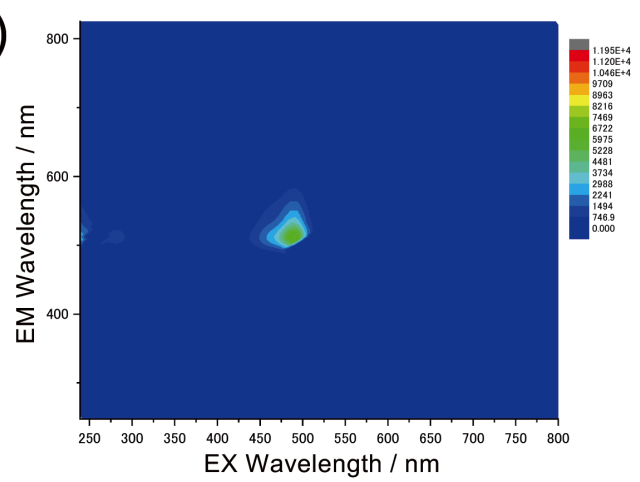

(b)

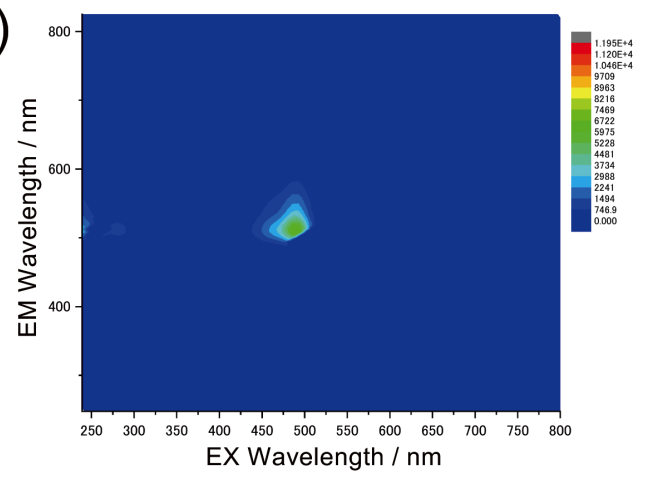

(d)

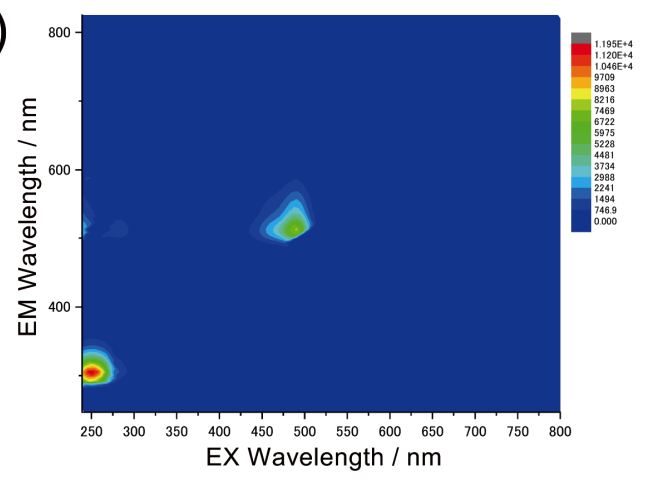

(C)

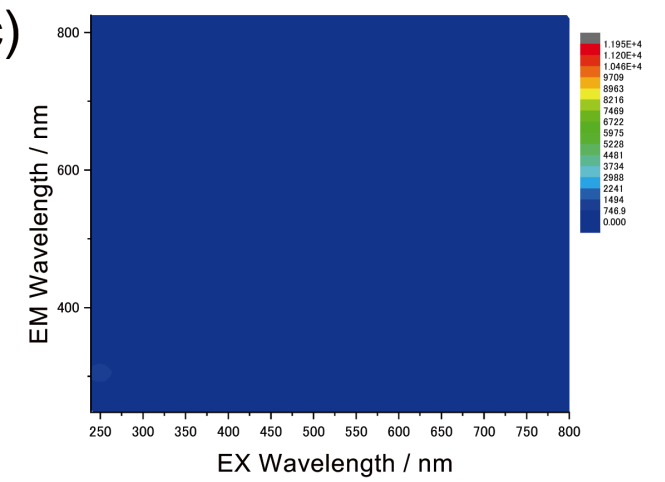

(e)

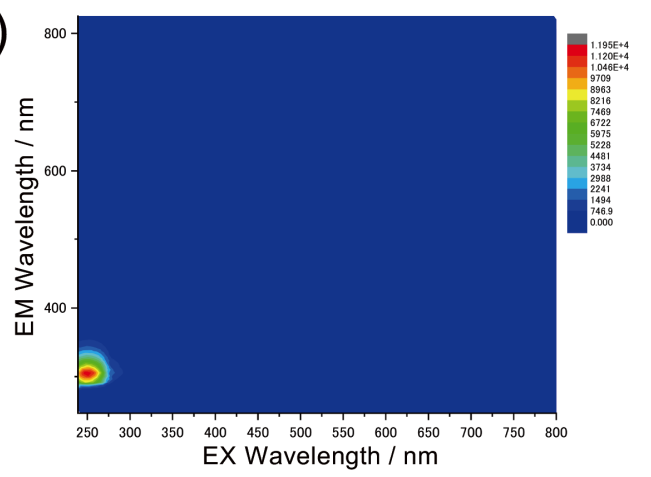

Fig. 3 3D EEM fluorescence spectra for Fluorescein, measured for a $50 \mathrm{nM}$ Fluorescein, (a); a $50 \mathrm{nM}$ Fluorescein $+10^{5}$-times diluted LPS32 solution, (b); a 105-times diluted LPS32 solution, (c); a $50 \mathrm{nM}$ Fluorescein $+10^{4}$-times diluted LPS32 solution, (d); and a 104-times diluted LPS32 solution, (e). All of the solutions prepared contained $0.01 \%$ Tween $20+1$ X D-PBS. Each spectrum mentioned here is an average of three (a) or five (b-e) independently-observed spectra.

Table 1 Excitation and emission wavelengths as well as fluorescent intensities for Fluorescein, Methylene Blue, and Rhodamine 6G (Each wavelength is that showing the maximum fluorescent intensity in Figs. 3, 5, and 6)

\begin{tabular}{llccc}
\hline & & Only dye & Dye $+10^{4}$-times dilutes LPS32 & Dye + 105-times dilutes LPS32 \\
\hline \multirow{2}{*}{ Fluorescein } & Excitation/nm & 485 & 488 & 488 \\
& Emission/nm & 513 & 508 & 513 \\
Intensity (arb. unit) & $6479 \pm 616$ & $6758 \pm 300$ & $7586 \pm 321$ \\
& Excitation/nm & 682 & 665 & 668 \\
Rethylene Blue & Emission/nm & $362 \pm 21$ & $428 \pm 23$ & 687 \\
& Intensity (arb. unit) & 527 & 527 & $421 \pm 16$ \\
& Excitation/nm & 550 & 550 & 527 \\
& Emission/nm & $9676 \pm 586$ & $9757 \pm 663$ \\
\hline
\end{tabular}

increase in the fluorescent intensity.

With the use of Rhodamine $6 \mathrm{G}$, on the other hand, we observed different results in 3D EEM fluorescence spectroscopic measurements (Fig. 6). The figure shows an excitation wavelength of $527 \mathrm{~nm}$ and an emission wavelength of $550 \mathrm{~nm}$. The fluorescent intensity was $9305 \mathrm{AU}$. Adding 105-times diluted LPS32 particles to this solution did not change the excitation or emission wavelengths. Adding the $10^{4}$-times 
(a)

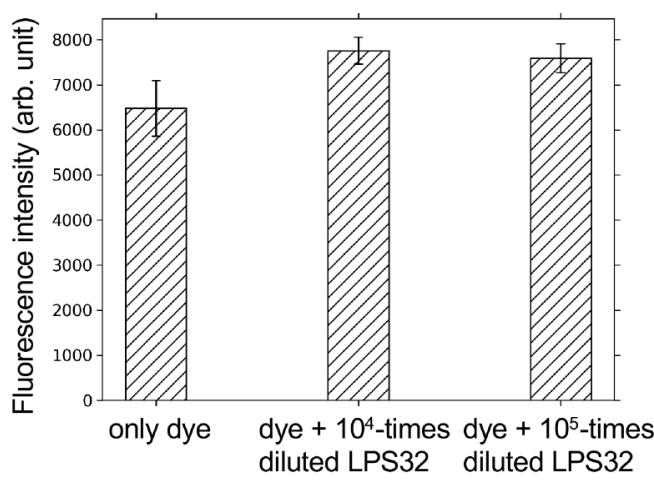

(b)

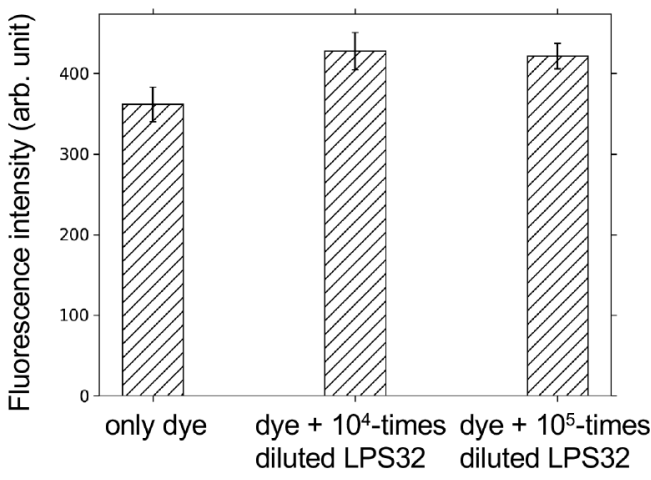

(c)

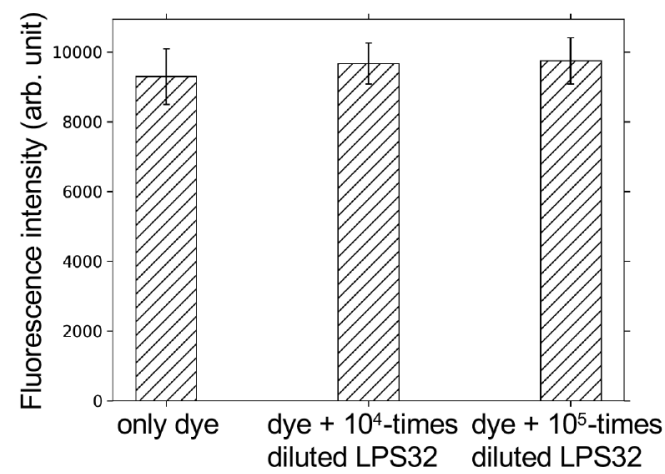

Fig. 4 Comparison of the fluorescent intensity for $50 \mathrm{nM}$ Fluorescein, $50 \mathrm{nM}$ Fluorescein $+10^{4}$-times diluted LPS32, and $50 \mathrm{nM}$ Fluorescein $+10^{5}$-times diluted LPS32, (a); for $50 \mathrm{nM}$ Methylene Blue, $50 \mathrm{nM}$ Methylene Blue + 104-times diluted LPS32, and $50 \mathrm{nM}$ Methylene Blue $+10^{5}$-times diluted LPS32, (b); and for $50 \mathrm{nM}$ Rhodamine $6 \mathrm{G}, 50 \mathrm{nM}$ Rhodamine $6 \mathrm{G}+10^{4}$-times diluted LPS32, and $50 \mathrm{nM}$ Rhodamine $6 \mathrm{G}+10^{5}$-times diluted LPS32, (c). All of the prepared solutions contained $0.01 \%$ Tween $20+1$ X D-PBS.

diluted LPS32 particles showed only a statistically insignificant $4.00 \%$ increase. The Welch's $t$-test also supports this result $(p=0.48)$. These results are probably because Rhodamine 6G is not much adsorbed to the surface of polystyrene, or the fluorescent properties are not significantly changed even if Rhodamine $6 \mathrm{G}$ is adsorbed.

The observed changes in the fluorescent intensity that result from the interacts between fluorescent dyes and polystyrene appear to be related to the affinity of the dyes to polystyrene and the structural stability of the dyes. Alternatively, it may be due to the environment around the dyes changing from a hydrophilic environment of the bulk of the aqueous solution to a hydrophobic environment of the surface of the polystyrene. Enhancements of the fluorescent intensity are due to several mechanisms, including localized surface plasmon resonance, ${ }^{24}$ solvato- chromism, ${ }^{25}$ and so on. Further studies are needed to fully understand the phenomena studied here, but these results reveal that interactions between the dye and polystyrene changed the fluorescent properties, and that the utilization of this phenomenon has future potential to contribute to the development of techniques to identify the materials of which plastic particles are made. The use of the observed phenomena in a feasible plastic materials identification tool, however, needs more comprehensive and detailed investigations of this phenomenon using a wide range of plastic materials.

\section{Conclusions}

Based on 3D EEM fluorescence spectroscopy, which makes it possible to observe excitation wavelength-dependent emission spectra, we studied the relationship between the interaction of fluorescent dyes with polystyrene particles an well as the dyes Fluorescein, Methylene Blue, and Rhodamine 6G. Fluorescein and Methylene Blue showed increases in the fluorescent intensity, while Rhodamine $6 \mathrm{G}$ showed hardly any intensity changes. This is probably due to the affinity of the dyes to the polystyrene particle surface, the structural stability of the dyes on the surface, and the change in the environment around the dyes after their adsorption to the plastic surfaces. We conclude that we have shown the potential to look for appropriate fluorescent dyes using the method studied here to identify and estimate individual plastic materials.

However, these fluorescent dyes showed only an up to $19.7 \%$ increase in the fluorescent intensity (in the case of Fluorescein). The reason for this might be attributed to the dyes' weak affinity to the polystyrene surface. We are now exploring more hydrophobic fluorescent dyes with higher affinities to the polystyrene surface. The use of suitable solvents and surfactants might enhance their low solubilities in water. Further improvements to this method should allow the establishment of a method to enable in situ observations of changes in the fluorescent properties without any separation or filtration processes after the adsorption of the dyes to plastics.

New polymer materials are continuously being developed. There is growing research interest concerning biodegradable plastic materials from the viewpoint of reducing the environmental load, one of the United Nation's Sustainable Development Goals (SDGs). ${ }^{26}$ A new report, however, reveals the toxicity of biodegradable plastic particles to be almost the same as that of artificially synthesized plastic particles. ${ }^{17}$ According to this report, the toxicity of plastic particles derives from the adsorption of toxic chemicals to the particles. While the toxicity of chemicals is one of the important research areas,,$^{27,28}$ the affinity of plastic particles to toxic chemicals is also another important research area. We believe the method studied here will be able to contribute to the development of fluorescent dyes for identifying plastic particles with high affinity to toxic chemicals, including other fluorescent dyes targeting materials that remain undetected by conventional methods designed to identify individual plastics.

\section{Acknowledgements}

We are grateful to Ms. Satoko Furukawa of the Environmental Management Research Institute (EMRI), National Institute of Advanced Industrial Science and Technology (AIST) for her help to collect and process data for particle counting and fluorescent spectroscopy. We also appreciate Dr. Tetsuya 
(a)

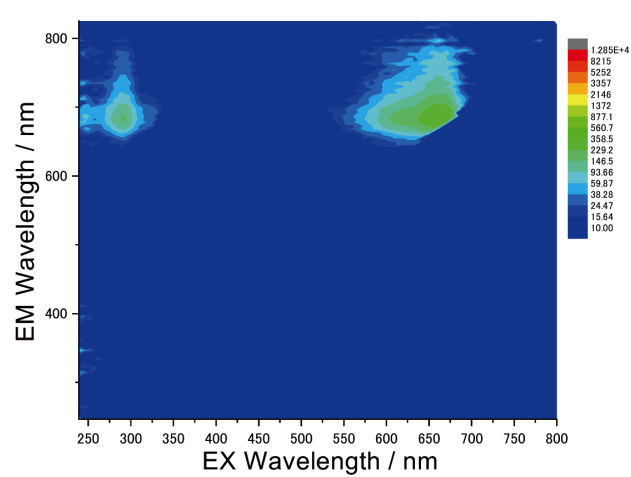

(b)

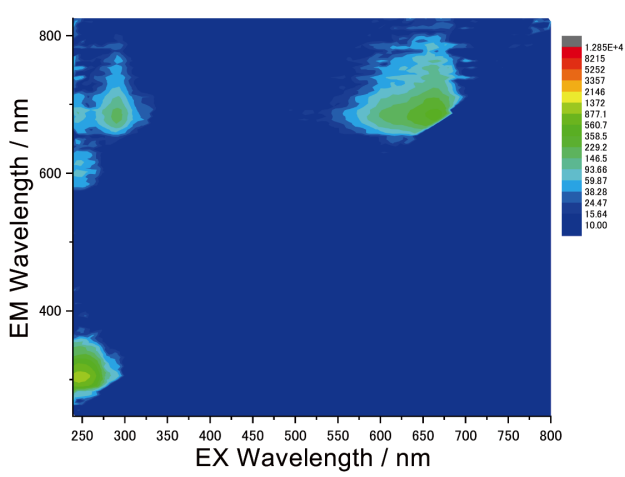

(d)

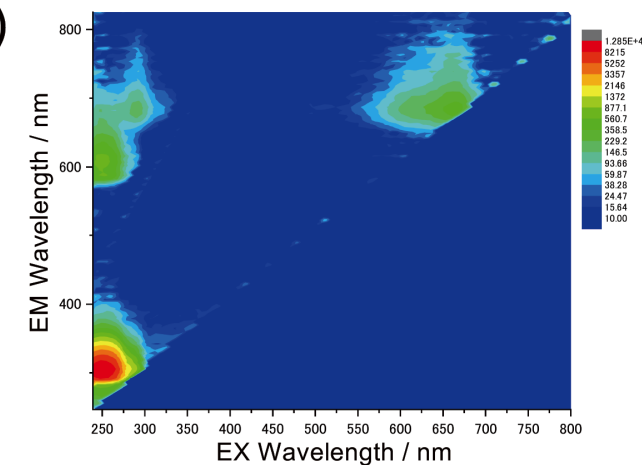

(c)

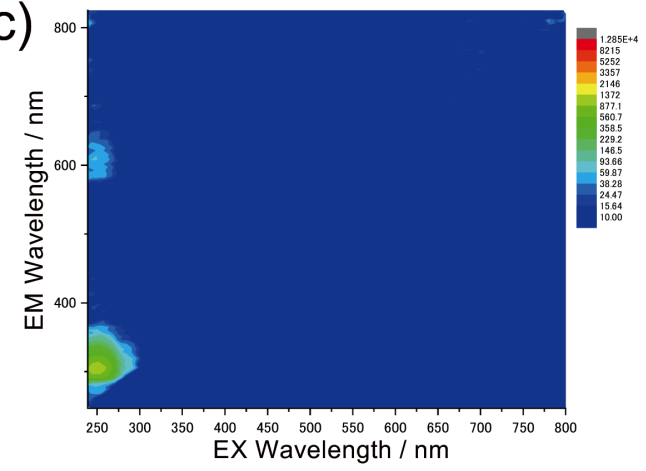

(e)

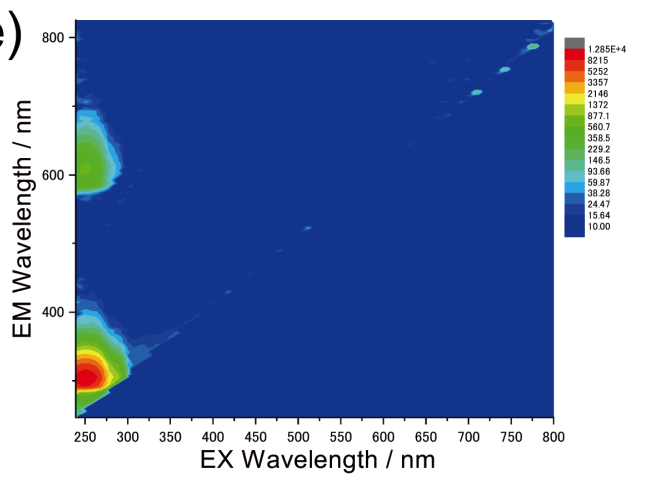

Fig. 5 3D EEM fluorescence spectra for Methylene Blue, measured for a $50 \mathrm{nM}$ Methylene Blue, (a); a $50 \mathrm{nM}$ Methylene Blue $+10^{5}$-times diluted LPS32 solution, (b); a 105-times diluted LPS32 solution, (c); a $50 \mathrm{nM}$ Methylene Blue $+10^{4}$-times diluted LPS32 solution, (d); and a 104-times diluted LPS32 solution, (e). All of the prepared solutions contained $0.01 \%$ Tween $20+1$ X D-PBS. Each spectrum described here is an average of three (a) or five $(b-e)$ independently-observed spectra.

Nakazato of EMRI, AIST, helping to give useful comments to the obtained results. This work was partially supported by a JSPS KAKENHI Grant Number JP19K05536 (awarded to HA) and by the River Fund of The River Foundation, Japan, Number 2020-5211-024 (awarded to HA).

\section{Supporting Information}

This material is available free of charge on the Web at http:// www.jsac.or.jp/analsci/.

\section{References}

1. CAS Registry-The Gold Standard for Chemical Substance Information, http://support.cas.org/content/chemical-substances (checked on November 22nd, 2020).

2. Z. Wang, G. W. Walker, D. C. G. Muir, and K. NagataniYoshida, Environ. Sci. Technol., 2020, 54, 2575.

3. M. S. Wolff, J. P. Buckley, S. M. Engel, R. S. McConnell, and D. B. Barr, Curr. Opin. Pediatr., 2017, 29, 218.

4. A. R. Zota, V. Singla, G. Adamkiewicz, S. D. Mitro, and R. E. Dodson, J. Epidemiol. Community Health, 2017, 71, 937.

5. W. J. Shim and R. C. Thomposon, Arch. Environ. Contam. Toxicol., 2015, 69, 215.

6. E. L. Teuten, J. M. Saquing, D. R. U. Knappe, M. A. Barlaz, S. Jonsson, A. Björn, S. J. Rowland, R. C. Thompson, T. S. Galloway, R. Yamashita, D. Ochi, Y. Watanuki, C. Moore, P. H. Viet, T. S. Tana, M. Prudente, R. Boonyatumanond, M. P. Zakaria, K. Akkhavong, Y. Ogata, H. Hirai, S. Iwasa, K. Mizukawa, Y. Hagino, A. Imamura, M. Saha, and H. Takada, Phil. Trans. R. Soc. B, 2009, 364, 2027.

7. O. Setälä, V. Fleming-Lehtinen, and M. Lehtiniemi, Environ. Pollut., 2014, 185, 77.

8. L. S. Fendall and M. A. Sewell, Mar. Pollut. Bull., 2009, 58,1225 .

9. C. M. Rochman, S. M. Kross, J. B. Armstrong, M. T. Bogan, E. S. Darling, S. J. Green, A. R. Smyth, and D. Veríssimo, Environ. Sci. Technol., 2015, 49, 10759. 
(a)

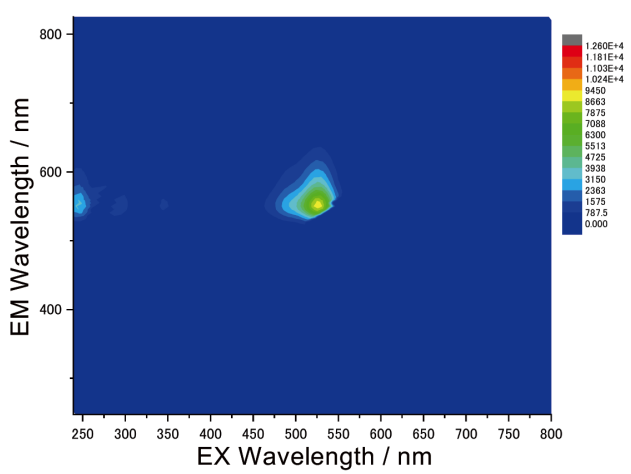

(b)

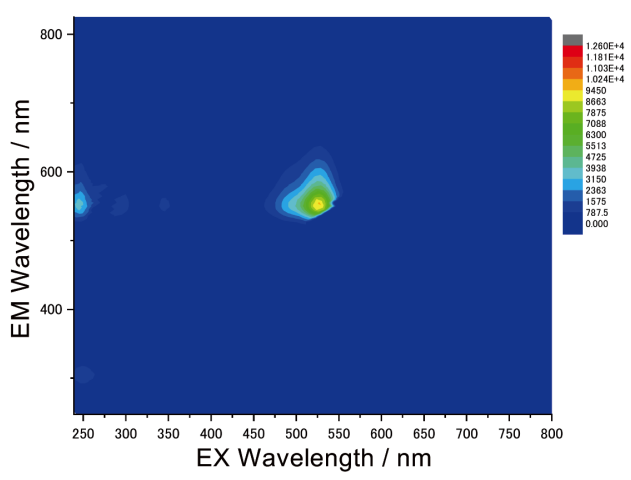

(d)

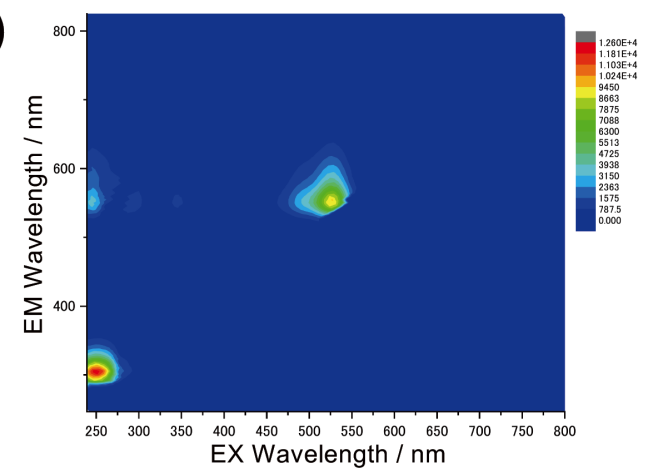

(c)

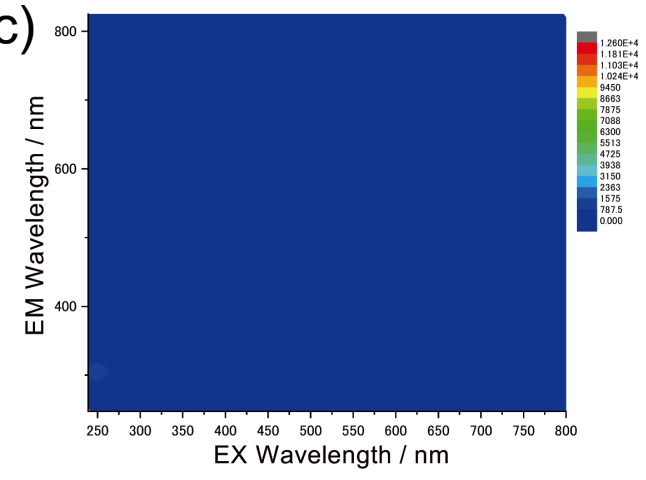

(e)

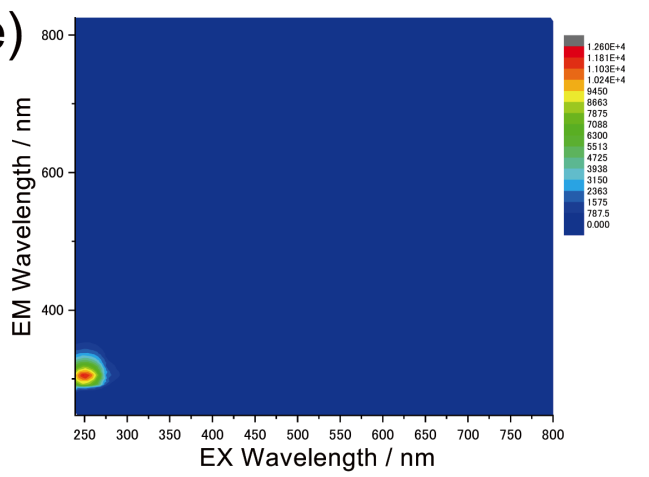

Fig. 6 3D EEM fluorescence spectra for Rhodamine 6G, measured for a $50 \mathrm{nM}$ Rhodamine 6G, (a); a $50 \mathrm{nM}$ Rhodamine $6 \mathrm{G}+10^{5}$-times diluted LPS32 solution, (b); a 105-times diluted LPS32 solution, (c); a $50 \mathrm{nM}$ Rhodamine 6G + 104-times diluted LPS32 solution, (d); and a 104-times diluted LPS32 solution, (e). All of the prepared solutions contained $0.01 \%$ Tween $20+1$ X D-PBS. Each spectrum described here is an average of three (a) or five $(b-e)$ independently-observed spectra.

10. M. A. Browne, P. Crump, S. J. Niven, E. Teuten, A. Tonkin, T. Galloway, and R. Thompson, Environ. Sci. Technol., 2011, 45, 9175.

11. A. L. Andrady, Mar. Pollut. Bull., 2011, 62, 1596.

12. J. Ha and M.-K. Yeo, Mol. Cell. Toxicol., 2018, 14, 353.

13. J. C. Prata, V. Reis, J. T. V. Matos, J. P. da Costa, A. C. Duarte, and T. Rocha-Santos, Sci. Total Environ., 2019, 690, 1277.

14. W. J. Shim, Y. K. Song, S. H. Hong, and M. Jang, Mar. Pollut. Bull., 2016, 113, 469.

15. G. Erni-Cassola, M. I. Gibson, R. C. Thompson, and J. A. Christie-Oleza, Environ. Sci. Technol., 2017, 51, 13641.

16. K. J. Wiggin and E. B. Holland, Mar. Pollut. Bull., 2019, $143,152$.

17. L. Zimmermann, A. Dombrowski, C. Völker, and M. Wagner, Environ. Int., 2020, 145, 106066.

18. T. Maes, R. Jessop, N. Wellner, K. Haupt, and A. G. Mayes, Sci. Rep., 2017, 7, 44501.

19. H. Tong, Q. Jiang, X. Zhong, and X. Hu, Environ. Sci.
Pollut. Res., 2021, 28, 4209.

20. S. Tyagi and F. R. Kramer, Nat. Biotechnol., 1996, 14, 303.

21. S. Tyagi, D. P. Bratu, and F. R. Kramer, Nat. Biotechnol., 1998, 16, 49.

22. D. M. Kolpashchikov, Scientifica, 2012, 2012, 928783.

23. H. Zipper, H. Brunner, J. Bernhagen, and F. Vitzthum, Nucleic Acids Res., 2004, 32, e103.

24. J. R. Lakowicz, Anal. Biochem., 2005, 337, 171.

25. A. Marini, A. Muñoz-Losa, A. Biancardi, and B. Mennucci, J. Phys. Chem. B, 2010, 114, 17128.

26. United Nations Development Programme, World leaders adopt Sustainable Development Goals, http://www.undp. org/content/undp/en/home/presscenter/pressreleases/2015/ 09/24/undp-welcomes-adoption-of-sustainable-developmentgoals-by-world-leaders.html (checked on November 22nd, 2020).

27. M. Poteser, Biomonitoring, 2017, 4, 11 .

28. H. Aoki, H. Tani, K. Nakamura, H. Sato, M. Torimura, and T. Nakazato, Toxicol. Appl. Pharmacol., 2020, 392, 114929. 